\title{
CONSTANT SURFACE HEATING OF A VARIABLE CONDUCTIVITY HALF-SPACE*
}

BY

LEONARD Y. COOPER

Bell T'elephone Iaboratories, Incorporated, Whippany, New Jersey

\begin{abstract}
A solution to the problem of constant surface heating of an initially constant-temperature, $T_{0}^{*}$, half-space where the material in question has a temperaturedependent thermal conductivity is obtained. The thermal conductivity, $k^{*}$, is specifically given by $k^{*}=k_{0}^{*} \exp \left[\lambda\left(T^{*}-T_{0}^{*}\right) / T_{0}^{*}\right]$. The solution is valid for both heating and cooling of the material where $\lambda$ and $k_{0}^{*}$ are arbitrary in magnitude, and $\lambda$ can be either positive or negative in sign.

The technique of solution of the nonlinear problem considered here is an extension of an integration method used for the boundary layer equations of fluid mechanics. The present success and generality of the technique suggest its use for the solution of more complicated variable property transient heat conduction problems.
\end{abstract}

1. Introduction. Most problems in unsteady heat conduction can be mathematically described by boundary value problems of the linear theory of diffusion. It often happens, however, that such phenomena elude the convenience of such a classical analysis. This study is concerned with the possible application of integration techniques, successfully used in the nonlinear problems of boundary layer theory in fluid mechanics, to nonlinear problems of unsteady heat conduction (and, of course, other diffusion phenomena). In particular, the present work treats a specific "test" boundary value problem in the diffusion of heat and incorporates in its solution the integration technique of Meksyn [1] as applied to boundary layer theory.

The specific problem under consideration is the constant surface heating, $H^{*}$, of an initially constant-temperature half-space where the dimensionless thermal conductivity, $\Phi$, is given by $\exp [\lambda \theta]$. Here $\theta=\left(T^{*}-T_{0}^{*}\right) / T_{0}^{*}$ is a dimensionless temperature, $T^{*}$ being the initial temperature of the medium. ${ }^{1}$

Transient heat conduction through such a variable conductivity material half-space has been previously considered for conditions of imposed constant surface temperatures rather than constant heat transfer rates. Crank [2] discusses the solution to this problem at length. As it happens, this problem has a similarity variable $\eta \sim x t^{-1 / 2}(x$ and $t$ are dimensionless distance into the slab and time respectively). Thus the second order partial differential equation for, say, $\Phi$ can be reduced to an ordinary differential equation. This state of affairs is analogous to the boundary layer problem for flow over a flat plate.

For the present problem, however, no such reduction is possible. Here the situation is analogous to, say, flow over a flat plate with suction. Nevertheless, as in boundary layer theory, it is useful to pose the problem in terms of the independent variables $\eta$ and $t$ (we will use $\zeta \sim t^{1 / 2}$ ). Using these variables the resulting boundary value problem

* Received February 15, 1968. This work was supported by the U. S. Army under Contract DA-30069-AMC-333(Y).

'Starred and unstarred quantities always refer to dimensional and dimensionless quantities respectively. 
governing $\Phi$ is such as to suggest an asymptotic form for large $\eta$ of $\Phi_{\eta}$ (obtained from a first integral in $\eta$ of the governing equation). This form of $\Phi_{\eta}$ is found to be $\Psi(\eta, \zeta) \exp$ $\left[-\eta^{2} / 2\right]$ where, compared to $\exp \left[-\eta^{2} / 2\right], \Psi$ is a slowly changing function in $\eta$. The idea of Meksyn incorporated here is (a) to represent $\Psi$ by its Taylor expansion about $\eta=0$, (b) to perform the integration of $\Phi_{\eta}$ as represented in the above form out to $\eta \rightarrow \infty$, and (c) to apply to the resulting representation for $\Phi(\infty, \zeta)$ the boundary condition required by the solution function $\Phi$ at $\eta \rightarrow \infty$. The application of this procedure results in equations involving slowly convergent or divergent infinite series which are rendered (more rapidly) convergent by use of the Euler transformation [1], [3]. The solutions to these equations eventually yield results for the coefficients of the double expansion of the solution function $\Phi$ about $(\eta, \zeta)=(0,0)$.

2. Formulation of the problem. Consider an isothermal body of temperature $T_{0}^{*}$ filling the half-space $x^{*} \geq 0$ where the material is such that the density, $\rho^{*}$, and specific heat, $C^{*}$, are both constant while the thermal conductivity, $k^{*}$, is a function of temperature, $T^{*}$, and is specifically given by

$$
k^{*}\left(T^{*}\right)=k_{0}^{*} \exp \left[\lambda\left(T^{*}-T_{0}^{*}\right) / T_{0}^{*}\right]
$$

where $k_{0}^{*}$ is the conductivity at temperature $T_{0}^{*}$ and $\lambda$ is arbitrary both in magnitude and sign. At an initial time, $t^{*}=0$, the surface of the body, $x^{*}=0$, is exposed to a constant and uniform heat flux, $H^{*}$ (of arbitrary sign), while no other heat sources throughout $x^{*}>0$ are allowed.

To obtain the temperature history, $T^{*}$, of the above material as a function of $x^{*}$ and $t^{*}$, we define the following dimensionless variables along with a dimensionless heat flux:

$$
\begin{aligned}
& x=x^{*}\left(T_{0}^{*} C^{*^{3}}\right)^{1 / 2} \rho^{*} / k_{0}^{*} ; \quad t=t^{*} T_{0}^{*} C^{*^{2}} \rho^{*} / k_{0}^{*} \\
& \theta=\left(T^{*}-T_{0}^{*}\right) / T_{0}^{*} ; \quad \Phi=k^{*} / k_{0}^{*} ; \quad H=H^{*} /\left[\left(T_{0}^{*} C^{*}\right)^{3 / 2} \rho^{*}\right] .
\end{aligned}
$$

The pertinent boundary value problems which must be solved either for the dimensionless temperature, $\theta(x, t)$, or the dimensionless conductivity, $\Phi(x, t)$, then take the form

$$
\begin{array}{rlcc}
\theta_{x x}+\lambda\left(\theta_{x}\right)^{2}=\theta_{t} \exp [-\lambda \theta] & \text { or } & \Phi \Phi_{x x}=\Phi_{t} ; x, t \geq 0 \\
\theta_{x}(0, t)=-H \exp [-\lambda \theta(0, t)] & \text { or } & \Phi_{x}(0, t)=-\lambda H ; t>0 \\
\theta(x, 0)=0 & \text { or } & \Phi(x, 0)=1 ; x>0 \\
\lim _{x \rightarrow \infty ; t \text { fixed }} \theta(x, t)=0 & \text { or } & \lim _{x \rightarrow \infty ; t \text { ixed }} \Phi(x, t)=1 .
\end{array}
$$

Clearly the above formulations are valid for the constant-conductivity case, $\lambda=0$. We will consider here the case $\lambda \neq 0$. If the solution can be obtained, we would expect that taking the limit as $\lambda \rightarrow 0$ would yield the well-known constant-conductivity solution [4].

We now define new variables $\eta, \zeta$, and $V$ as

$$
\eta=x /(2 t)^{1 / 2} ; \quad \zeta=-\lambda H(2 t)^{1 / 2} ; \quad V=\lambda \theta .
$$

Then the problem for $\Phi$ becomes

$$
\begin{gathered}
\Phi \Phi_{\eta \eta}+\eta \Phi_{\eta}=\zeta \Phi_{\zeta} \\
\lim _{\eta \rightarrow 0 ; \zeta \text { fixed }} \Phi_{\eta}=\zeta ; \quad \lim _{\eta \rightarrow \infty ; \zeta \text { fixed }} \Phi=1 .
\end{gathered}
$$


We will seek a solution to the latter of these problems. The solution for the new dimensionless temperature $V$ can then be obtained from the simple relationship

$$
V=\ln \Phi \text {. }
$$

3. The solution. The solution of the problem of (5) and (6) incorporates techniques of Meksyn originally suggested for the general two-dimensional boundary layer equations of fluid mechanics.

Basically, the technique of solution is one of using an expansion in $\eta$ and $\zeta$ of the dependent variable $\Phi$. The boundary condition at the body surface (the first condition of (6)) is automatically incorporated in such an assumed expansion. The important contribution of the Meksyn solution technique utilized here is the integration over $\eta$ of $\Phi_{\eta}$, obtained from a first integral of (5), where this function is represented in a "quasiasymptotic" form. As will be seen, this allows for a definite representation of $\Phi(\infty, \zeta)$ (in terms of a slowly convergent or divergent infinite series of functions of $\zeta$ ) from which some meaning can be extracted by use of the Euler transformation.

We assume that $\Phi$ has an expansion sbout $\eta=0$ which has some "reasonable" radius of convergence (possibly $\zeta$-dependent) in the $\eta$ plane. Thus we assume

$$
\Phi=\sum_{n=0}^{\infty} a_{n}(\zeta) \eta^{n}
$$

where $a_{1}(\zeta)=\zeta$. Note that this assumed solution form automatically satisfies the first boundary condition of $(6)$.

Taking the above solution form and using it in (5), we equate coefficients of like powers of $\eta$, thereby obtaining solutions for the $a_{n}(\zeta)$ as functions of $a_{0}(\zeta)$ through the following recursion relations: For $n \geq 2$,

$$
\begin{aligned}
a_{n}(\zeta)=\left[n(n-1) a_{0}(\zeta)\right]^{-1} & {\left[\zeta a_{n-2}^{\prime}(\zeta)-(n-2) a_{n-2}(\zeta)+n(n-2)\left\{1+(-1)^{n}\right\} a_{R(n)}^{2}(\zeta) / 8\right.} \\
& \left.-\sum_{i=1}^{R(n)}\{(n-j)(n-j-1)+j(j-1)\} a_{j}(\zeta) a_{n-j}(\zeta)\right]
\end{aligned}
$$

where

$$
\begin{aligned}
& a_{n}^{\prime}(\zeta) \equiv \frac{d}{d \zeta} a_{n}(\zeta) \\
& a_{1}(\zeta) \equiv \zeta
\end{aligned} ; \quad R(n) \equiv \begin{cases}n / 2 & \text { for } n \text { evelı } \\
(n-1) / 2 & \text { for } n \text { odd } .\end{cases}
$$

It should be noted from the above that $a_{n}(0)=0, n \geq 1$.

We now expand $a_{0}(\zeta)$ about $\zeta=0$.

$$
a_{0}(\zeta)=1+\sum_{n=1}^{\infty} \alpha_{n} \zeta^{n} .
$$

The leading term of 1 in the above expansion allows continuity of $\Phi$ at $(\eta, \zeta)=(0,0)$. We assume that such continuity, not required by the boundary conditions, is a feature of the solution. In view of our expansion for $\Phi$ and the recursion relationships for the $a_{n}(\zeta)$, a solution for $a_{0}(\zeta)$, i.e., the Taylor coefficients, $\alpha_{m}$, of $a_{0}(\zeta)$, would complete the formal solution to our boundary value problem. It is the application of the remaining boundary condition of (6), of course, that will eventually determine those constants.

From the second condition of (6) it is clear that $\Phi_{\eta} \rightarrow 0$ and $\Phi_{\zeta} \rightarrow 0$ for $\eta \rightarrow \infty$. If we 
assume that $\Phi_{\zeta}=O\left(\Phi_{\eta}\right)$ or at least $\Phi_{\zeta}=o\left(\eta \Phi_{\eta}\right)$ for $\eta \rightarrow \infty$, then for large $\eta$ the asymptotic form for a first integral of (5) will be $\Phi_{\eta} \sim \Psi(\eta, \zeta)$ exp $\left[-\eta^{2} / 2\right]$, where for large $\eta, \Psi$ is slowly changing compared to $\exp \left[-\eta^{2} / 2\right]$. We therefore take the form for $\Phi_{\eta}$ as

$$
\Phi_{\eta}=\Psi(\eta, \zeta) \exp \left[-\eta^{2} / 2\right]
$$

In consonance with the above equality sign the function $\Psi$ will be represented according to its expansion about $\eta=0$ rather than in an asymptotic form. With $\Phi_{\eta}$ taken in this "quasi-asymptotic" form it will easily be possible to perform a second integration over $\eta$ thereby obtaining an equation for $\Phi$. Moreover, to this resulting equation for $\Phi$, valid within the radius of convergence of $\Psi$ and in some sense representitive of $\Phi$ outside such a region, we will be able to directly apply the second condition of (6) at $\eta \rightarrow \infty$.

We now expand $\Psi(\eta, \zeta)$ about $\eta=0$. For later convenience we take the following form:

$$
\Psi(\eta, \zeta)=\sum_{n=0}^{\infty}\left[2^{(1-n) / 2} g_{n}(\zeta) / \Gamma\{(n+1) / 2\}\right] \eta^{n}
$$

where $\Gamma(x)$ is the gamma function. Substituting this above expansion for $\Psi$ and the expansion of (8) for $\Phi$ into the right- and left-hand sides of Eq. (11) respectively, we expand $\exp \left[-\eta^{2} / 2\right]$ about $\eta=0$, equate like powers of $\eta$ and eventually obtain the following solution for the $g_{n}(\zeta)$ in terms of the $a_{n}(\zeta)$ :

$$
g_{n}(\zeta)=2^{(n-1) / 2} \Gamma\{(n+1) / 2\} \sum_{p=0}^{R(n)}(n-2 p+1) a_{n-2 p+1}(\zeta) /\left(2^{p} p !\right) ; \quad n \geq 0
$$

where $R(n)$ is defined after Eq. (9). Note that for all $n, g_{n}(0)=0$.

We now use our expansion (12) for $\Psi$ in the first integral expression of (11) and perform another integration over the variable $\eta$. This results in

$$
\Phi(\eta, \zeta)=\int_{0}^{\eta} \sum_{n=0}^{\infty}\left[2^{(1-n) / 2} g_{n}(\zeta) / \Gamma\{(n+1) / 2\}\right] \exp \left(-\mu^{2} / 2\right) \mu^{n} d \mu+a_{1}(\zeta)
$$

or, interchanging integration and summation,

$$
\Phi(\eta, \zeta) \sim \sum_{n=0}^{\infty} g_{n}(\zeta) P^{2}\left\{(n+1) / 2, \eta^{2} / 2\right\}+a_{0}(\zeta)
$$

where $P(x, y)$ is the normalized incomplete gamma function

$$
P(x, y)=\Gamma^{-1}(x) \int_{0}^{0} e^{-\mu} \mu^{x-1} d \mu .
$$

It should be noticed that the exchange of integration and summation above would only be valid for $\eta$ within the radius of convergence (in the $\eta$ plane) of the expansion of $\Psi$, and that in general the results of this exchange of operation will yield a relationship for $\Phi$ that is not necessarily an equality. Thus for $\eta \rightarrow \infty$, a case that will be of particular interest here, the quantity on the right-hand side of (14) can at best be considered as representitive of the function $\Phi$.

We are now in a position formally to satisfy the remaining boundary condition of (6). Doing so with the use of Eq. (14), we obtain

$$
\sum_{n=0}^{\infty} g_{n}(\zeta) \sim 1-a_{0}(\zeta)
$$


By considering the Taylor expansions of the $g_{n}(\zeta)$ and $a_{0}(\zeta)$ about $\zeta=0$ we would hope to relate coefficients of $\zeta^{m}$ in the above expression, thereby obtaining equations for the Taylor coefficients, $\alpha_{m}$, of $a_{0}(\zeta)$ and completing the solution. To this end we make the following definitions:

$$
a_{n}(\zeta)=\sum_{m=0}^{\infty} A_{n, m} \zeta^{m} ; \quad g_{n}(\zeta)=\sum_{m=1}^{\infty} G_{n, m} \zeta^{m} ; \quad n \geq 0 .
$$

Using the above definitions for the $A_{n, m}$ in the recursion relations (9) for the $a_{n}(\zeta)$ along with the definitions of $a_{0}(\zeta)$ and $a_{1}(\zeta)$, we find that the $A_{n, m}$ and, as a consequence of (13), the $G_{n, m}$ can be expressed as

$$
A_{n, m}=\mathcal{C}_{n, m}^{\prime} \alpha_{m}+F_{n, m}^{\prime} ; \quad G_{n, m}=\mathfrak{C}_{n, m} \alpha_{m}+\mathcal{F}_{n, m}
$$

where the $\mathfrak{C}_{n, m}^{\prime}$ and $\mathfrak{C}_{n, m}$ are constants and the $\mathscr{F}_{n, m}^{\prime}$ and $\mathcal{F}_{n, m}$ are polynomial functions of the $\alpha_{p}, p<m$. The solution for these are specifically found to be

$$
\begin{aligned}
& \mathfrak{C}_{0, m}^{\prime}=1, \quad m \geq 1 ; \quad \text { all } \quad \mathfrak{e}_{n, 0}^{\prime}=0 ; \quad \text { all } \quad \mathfrak{e}_{1, m}^{\prime}=0 ; \\
& \mathfrak{C}_{n, m}^{\prime}=(m-n+2) \mathfrak{C}_{n-2, m}^{\prime} /\{n(n-1)\}, \quad n \geq 2, \quad m \geq 1 \text {; } \\
& \mathcal{F}_{0,0}^{\prime}=1, \quad \text { all other } \quad \mathscr{F}_{n, 0}^{\prime}=0, \quad \text { all other } \quad \mathscr{F}_{0, m}^{\prime}=0 \text {; } \\
& \mathfrak{F}_{1,1}^{\prime}=1, \quad \text { all other } \mathscr{F}_{1, m}^{\prime}=0, \quad \text { all other } \quad \mathscr{F}_{n, 1}^{\prime}=0 \text {; } \\
& \mathcal{F}_{n, m}^{\prime}=(m-n+2) \mathcal{F}_{n-2, m}^{\prime} /\{n(n-1)\} \\
& -\sum_{p=1}^{m-1}\left[A_{n, p} \alpha_{m-p}-(n-2)\left\{1+(-1)^{n}\right\} A_{R(n), p} A_{R(n), m-p} /\{8(n-1)\}\right. \\
& \left.+\sum_{j=1}^{R(n)}\{(n-j)(n-j-1)+j(j-1)\} A_{i, p} A_{n-i, m-p} /\{n(n-1)\}\right], n, m \geq 2 ;
\end{aligned}
$$

$\left\{\begin{array}{l}\mathfrak{C}_{n, m} \\ \mathfrak{F}_{n, m}\end{array}\right\}=2^{(n-1) / 2} \Gamma\{(n+1) / 2\} \sum_{p=0}^{R(n)}\left\{(n-2 p+1) /\left(2^{p} p !\right)\right\}\left\{\begin{array}{l}\left.\mathfrak{C}_{n-2 p+1, m}^{\prime}\right\} \\ \mathcal{F}_{n-2 \mathfrak{p}+1, m}^{\prime}\end{array}\right\}$

Having expanded the $g_{n}(\zeta)$ according to $(16)$ and $a_{0}(\zeta)$ according to (10), we group like powers of $\zeta$ in Eq. (15) and eventually obtain

$$
\sum_{n=0}^{\infty} G_{n, m} \sim-\alpha_{m} ; \quad m \geq 1 .
$$

Thus, if the above sums have any meaning, e.g. if they converge, we would expect to have a set of recursion relationships for each $\alpha_{m}$ in terms of the $\alpha_{n}, n<m$. Moreover, these relationships would be linear in the unknown $\alpha_{m}$ of interest. Actually, as will be seen, the above sums appear to be either very slowly convergent (for $m=1$ ) or divergent. They can, however, be rendered convergent, or more rapidly convergent, by application of the Euler transformation. Meksyn makes extensive use of this transformation where the terms have known numerical values. Here, of course, the terms themselves contain the particular unknown, $\alpha_{m}$, being sought.

We will be interested in taking the Euler transformation of the columns of an array $\mathfrak{T}_{n, m}$. Such a transformation, designated here by the symbol $\varepsilon$, is defined by 


$$
\mathcal{E}\left(\mathfrak{T}_{n, m}\right) \equiv \mathcal{E}^{(1)}\left(\mathfrak{T}_{n, m}\right) \equiv 2^{-(n+1)} \sum_{a=0}^{n} \mathfrak{T}_{a, m}[n ! /\{(n-q) ! q !\}] .
$$

Repeated use of the Euler transformation is also of interest. Thus, for $p \geq 2$, we define

$$
\mathcal{E}^{(p)}\left(\mathfrak{T T}_{n, m}\right) \equiv \mathcal{E}\left\{\mathcal{E}^{(p-1)}\left(\mathfrak{T}_{n, m}\right)\right\}
$$

4. Calculation of the $\alpha_{m}$. We now proceed to calculate the Taylor coefficients, $\alpha_{m}$, of the expansion of the function $a_{0}(\zeta)$ for our problem. As, mentioned, we obtain these constants, one at a time, from the equations of (19) where the computation for a given $\alpha_{m}$ depends on all preceding $\alpha_{n}, n<m$. As it happens, the problem in such a computation is a problem of forcing convergence, or at least more rapid convergence, of the sum on the left-hand side of (19). To illustrate this point we proceed to calculate $\alpha_{1}$.

Using 9 terms in the series of (19) for $m=1$ we obtain

$$
\begin{aligned}
\sum_{n=0}^{\infty} G_{n, 1}=(\pi / 2)^{1 / 2} & +\alpha_{1}+(\pi / 2)^{1 / 2} / 2+2 \alpha_{1} / 3+3(\pi / 2)^{1 / 2} / 8+8 \alpha_{1} / 15 \\
& +5(\pi / 2)^{1 / 2} / 16+16 \alpha_{1} / 35+35(\pi / 2)^{1 / 2} / 128+\cdots \sim-\alpha_{1} .
\end{aligned}
$$

From observing the relative size of alternate terms it is clear that even if the above series was assumed to be convergent one could not expect a great deal of accuracy from an $\alpha_{1}$ calculated from the existing number of terms of the above equation. In any event we solve for $\alpha_{1}$ and obtain

$$
\alpha_{1} \stackrel{?}{=}-.84337087 \text {. }
$$

Hoping to render convergent or more rapidly convergent the above series, we apply the Euler transformation of (20) to the $G_{n, 1}$. This results in

$$
\begin{aligned}
\sum_{n=0}^{\infty} \varepsilon\left(G_{n, 1}\right)= & (\pi / 2)^{1 / 2} / 2+\left[(\pi / 2)^{1 / 2}+\alpha_{1}\right] / 4+\left[3(\pi / 2)^{1 / 2}+4 \alpha_{1}\right] / 16 \\
& +\left[15(\pi / 2)^{1 / 2}+22 \alpha_{1}\right] / 96+\left[35(\pi / 2)^{1 / 2} / 256+5 \alpha_{1} / 24\right] \\
& +\left[63(\pi / 2)^{1 / 2} / 512+183 \alpha_{1} / 960\right]+\left[693(\pi / 2)^{1 / 2} / 6144+169 \alpha_{1} / 960\right] \\
& +\left[429(\pi / 2)^{1 / 2} / 4096+4409 \alpha_{1} / 26880\right] \\
& +\left[6435(\pi / 2)^{1 / 2} / 65536+69 \alpha_{1} / 448\right]+\cdots \sim-\alpha_{1} .
\end{aligned}
$$

Solving the above for $\alpha_{1}$ we obtain

$$
\alpha_{1} \stackrel{?}{=}-.79782941 \text {. }
$$

Using this last value for $\alpha_{1}$ we evaluate the individual terms of $\varepsilon\left(G_{n, 1}\right)$ with the result

$$
\begin{aligned}
\sum_{n=0}^{\infty} \varepsilon\left(G_{n, 1}\right) \stackrel{?}{=}(\pi / 2)^{1 / 2}[1+.1877+.0568+.0208+.0082+ & .0034+.0015 \\
& +.0007+\cdots] .
\end{aligned}
$$

Assuming this series of terms to be convergent, the accuracy to the infinite sum, $\sum \varepsilon\left(G_{n, 1}\right)$, obtainable from the partial sum of a given number of terms appears to have been improved over the similar accuracy obtainable in (22). We conclude, then, that it is consistent to assume that the application of the Euler transformation to the series of Eq. (22) has resulted in forcing more rapid convergence of this infinite sum. The meaning 
of the word "consistent" is best understood in the context of the following recapitulation: (1) We have assumed that the Euler transformation forces convergence or more rapid convergence of the series of Eq. (22), and we have applied this transformation; (2) consistent with (1) we assumed that truncation of the resulting series of (24) after the ninth term will yield an accurate representation of its sum; (3) we solved for $\alpha_{1}$ in (24) based on these latter two assumptions; (4) using the calculated value for $\alpha_{1}$, we have shown that the terms of the above series, evaluated with this solution for $\alpha_{1}$ are consistent with our original assumption.

As it happens, $\alpha_{1}$ can be obtained exactly, e.g., from the solution to the problem of Eqs. (5) and (6) for $\eta=0$ in the limit $\zeta \rightarrow 0$. Such a limiting process leads to the solution for the surface temperature distribution for the problem where the material thermal conductivity in question is given as a constant. This well-known solution [4] dictates that

$$
\alpha_{1}=-(2 / \pi)^{1 / 2}=-0.797884561 \cdots \text {. }
$$

We have clearly obtained our approximate result for $\alpha_{1}$ in (25) to within $0.007 \%$ of its exact value. We would hope that using more terms in our series would increase our accuracy in this calculation. A computer computation for $\alpha_{1}$ using (24) with the series truncated to 34 terms has been performed. The result was a value for $\alpha_{1}$, compared to the above exact value, accurate to 12 significant figures.

Note that the value for $\alpha_{1}$ given by (23) and computed from the untransformed series of $(22)$ was $6 \%$ in error. That the result of that calculation should even be this accurate is due to the alternating signs of the terms of the original series. It is, in fact, this property of the original series that allows for the success of the Euler transformation [3].

In view of the above, it appears that there may be a significant improvement in accuracy of the sums represented in Eq. (19) after application of the Euler transformation. With this in mind we proceed to the calculation of $\alpha_{2}$.

Using the results of (17) and (18) and the exact value for $\alpha_{1}$ as given in (27), Eq. (19) for $m=2$ becomes

$$
\begin{aligned}
\sum_{n=0}^{\infty} G_{n, 2}=[0]+\left[2 \alpha_{2}-2 / \pi\right]+[1 / 2]+\left[2 \alpha_{2}-2 / \pi\right]+[1 / 4]+\left[2 \alpha_{2}-22 / 15 \pi\right] \\
+[1 / 4]+\left[2 \alpha_{2}-34 / 21 \pi\right]+[1 / 4]+\cdots \sim-\alpha_{2} .
\end{aligned}
$$

As it happens, the $\frac{1}{4}$ value of alternate terms continues to persist as more terms of the series are considered. For this reason the above series is clearly not convergent. We apply the Euler transformation to the $G_{n, 2}$. Then, using the 9 terms at our disposal, we solve the equation $\sum \mathcal{E}\left(G_{n, 2}\right)=-\alpha_{2}$ and obtain the result $\alpha_{2}=.12494787$. Inserting this value back into the series $\sum \mathcal{E}\left(G_{n, 2}\right)$ we obtain

$$
\sum_{n=0}^{\infty} \mathcal{E}\left(G_{n, 2}\right) \stackrel{?}{=}-.09668[0+1 .+.3535+.0303-.0505-.0375-.0116+.0025
$$

We assume that this series is convergent. Here, however, the terms are not monotonically decreasing as in the series of (26) for the computation of $\alpha_{1}$. There appears to be an oscillation of the terms about zero along with a decaying trend in their magnitude. Unfortunately, any definite conclusions here, with the few terms at our disposal, would be purely speculative. In view of this apparent oscillation of terms it is also difficult to ascertain the possible error in the value of $\alpha_{2}$ given above. 
A computer calculation for $\alpha_{2}$ from the equation $\sum \varepsilon\left(G_{n, 2}\right)=\alpha_{2}$ was obtained where the series was truncated to 50 terms and the exact value of $\alpha_{1}$ was used in the computation. The solution obtained was

$$
\alpha_{2}=\frac{1}{8}
$$

accurate to 16 significant figures. Using this solution the terms $\mathcal{E}\left(G_{n, 2}\right)$ are computed, the magnitude of the latter terms indicating that our accuracy for the value of $\alpha_{2}$ is only to 11 significant figures. In any event we assume that $\alpha_{2}$ is exactly given by $\alpha_{2}=\frac{1}{8}$, and this value will be used in further calculations. We mention that the oscillation about zero of the terms $\varepsilon\left(G_{n, 2}\right)$, along with a decaying trend in their magnitude with increasing $n$, as noted in our earlier calculation, continues to persist through $\mathcal{E}\left(G_{49,2}\right)$ of the present calculation.

The technique of applying the Euler transformation to the terms of an infinite sum in order to improve or possibly induce convergence is not necessarily successful. Especially when one deals with series whose properties are unknown, as in the present problem, it is only with the hindsight of the transformation results in hand that one can determine the success of the technique. And even then the determination of success or failure can generally only be argued in an heuristic fashion, e.g. our above "consistent" argument. Thus far we have used the Euler transformation in its simplest form. Namely, we have applied it only once to the various infinite series of Eq. (19), and we have applied it always starting with the first term, $G_{0, m}$. It is known that if the Euler transformation is successfully applied to a given series so as to, say, improve convergence of same then it is possible that applying the transformation a multiple number of times will further improve convergence. On the other hand, repeated transformations can slow down as well as speed up convergence. Moreover, for best results, the transformation need not necessarily be initiated with the first term of the series in question [1].

The single Euler transformation of the $G_{n, 2}$ starting from the first (zero) term appeared to be the optimum transformation for rendering $\sum G_{n, 2}$ most rapidly convergent; thus the above evaluation for $\alpha_{2}$. In considering the calculations for further $\alpha_{m}$ the various $\sum G_{n, m}, m \geq 3$, were made (more rapidly) convergent by consideration of multiple transformations of the type (21). Thus, only transformations initiated with the first term of these infinite sums were considered. In this way, the representations of (19) were changed to the following "apparent" equalities:

$$
\sum_{n=0}^{\infty} \varepsilon^{[p(m) 1}\left(G_{n, m}\right)=-\alpha_{m} .
$$

The solutions for the $\alpha_{m}, m \geq 3$ were obtained from the above equations with the infinite sums truncated to 58 terms. The results are

$$
\begin{aligned}
& \alpha_{3}=0.595407 \times 10^{-2} ; \quad \alpha_{4}=0.106534 \times 10^{-2} ; \quad \alpha_{5}=0.2655 \times 10^{-3} \\
& \alpha_{6}=0.780 \times 10^{-4} ; \quad \alpha_{7}=0.254 \times 10^{-4} ; \quad \alpha_{8}=0.88 \times 10^{-5} ; \\
& \alpha_{9}=0.32 \times 10^{-5} ; \quad \alpha_{10}=0.12 \times 10^{-5} ; \quad \alpha_{11}=0.5 \times 10^{-6} ; \quad \alpha_{12}=0.2 \times 10^{-6}
\end{aligned}
$$

where the significant figures shown for a given $\alpha_{m}$ are dictated by the order of magnitude of the higher order terms of $\varepsilon^{[p(m)]}\left(G_{n, m}\right)$. As for $m=2$, it should be noted that for $m \geq 3$ the $\mathcal{E}^{[\nu(m)]}\left(G_{n, m}\right)$ tend to oscillate about zero and decay in magnitude with increasing $n$. The function $p(m)$ up to $p(12)$ is given by 


$$
\begin{gathered}
p(1)=p(2)=1 ; \quad p(3)=p(4)=p(5)=p(6)=p(7)=p(8)=2 ; \\
p(9)=p(10)=p(11)=p(12)=3 .
\end{gathered}
$$

Presumably more accuracy could be obtained and more of the $\alpha_{m}$ could be evaluated if the sums of (29) would be approximated by a larger number of terms than used here.

5. Evaluation of $\Phi(\eta, \zeta)$. Using the expansions (10) and (16) of $a_{0}(\zeta)$ and $g_{n}(\zeta)$ respectively in (14), and grouping coefficients of like powers of $\zeta$, we obtain

$$
\Phi(\eta, \zeta) \sim 1+\sum_{m=1}^{\infty}\left\{\sum_{n=0}^{\infty} G_{n, m} P\left([n+1] / 2, \eta^{2} / 2\right)+\alpha_{m}\right\} \zeta^{m} .
$$

Note that, in view of (6), taking $\lim \eta \rightarrow \infty$ with $\zeta$ fixed reduces the above to (19) from which the $\alpha_{m}$ were eventually computed. It is therefore expected that, just as the series $\sum_{n=0}^{\infty} G_{n, m}, m \geq 1$ in (19) required Euler transformations in order to be rendered convergent, so our present sum $\sum G_{n, m} P\left([n+1] / 2, \eta^{2} / 2\right), \eta \neq 0$ will require similar transformations. We will therefore apply the multiple Euler transformation, $\mathcal{E}^{[p(m)]}$, to the terms of the internal sums of (32), thereby obtaining the following "apparent" equality:

$$
\Phi(\eta, \zeta)=1+\sum_{m=1}^{\infty}\left\{\sum_{n=0}^{\infty} \mathcal{E}^{[p(m)]}\left[G_{n, m} P\left([n+1) / 2, \eta^{2} / 2\right)\right]+\alpha_{m}\right\} \zeta^{m}
$$

In our work $\Phi$ has been evaluated according to (33) where the inner sum was truncated to 58 terms and the outer sum to 12 terms. In this computation the $\alpha_{m}$ are given by (27), (28) and (30) and the $G_{n, m}$ are computed from these according to (17) and (18). Moreover the $p(m)$ are given by (31). At least for $\eta=0$, it is estimated from the latter terms of the resulting finite series that the error in $\Phi$ from such a computation will be less than $5 \%$ provided $|\zeta|<1.8$, this error greatly diminishing as $|\zeta| \rightarrow 0$. The results of this computation are given in Fig. 1 where $\Phi$ is plotted as a function of $\zeta$ with $\eta$ as a parameter.

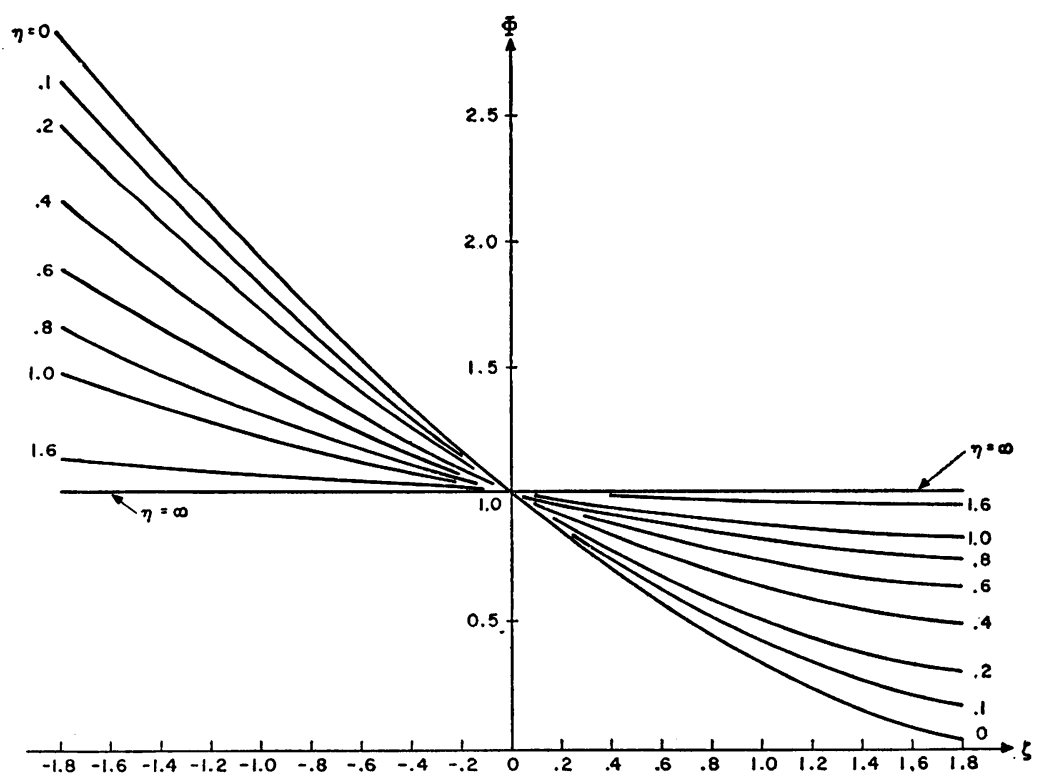

FIG. 1. The dimensionless conductivity, $\Phi$, as a function of $\zeta$ with $\eta$ as a parameter. 
Since the temperature history of the half-space as a function of time and depth was the specific goal of the originally posed boundary value problem, this has been obtained from our calculations for $\Phi$ and our definition (7) of the dimensionless temperature, $V$. This result is presented in Fig. 2 where $V$ is plotted as a function of $|\lambda H| x$ with $\lambda H|\lambda H| t$ as a parameter.

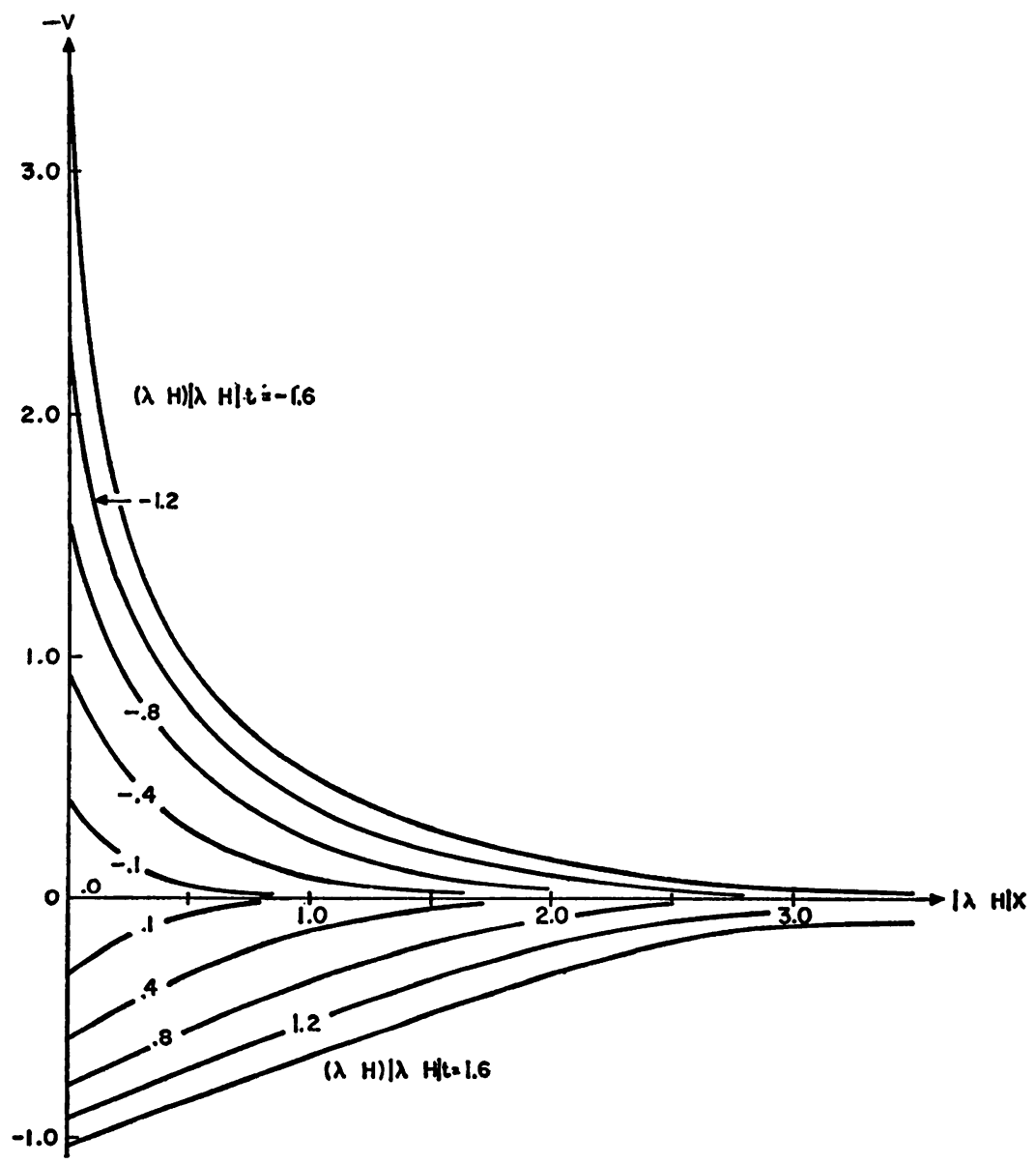

Fig. 2. The dimensionless temperature, $V$, as a function of $|\lambda H| x$ with $\lambda H|\lambda H| t$ as a parameter.

6. Results and conclusions. The physical phenomenon involved in heating the half-space under consideration is clearly seen in Fig. 2. This figure exhibits the intense surface-concentrated heating or cooling when $\lambda H<0$ as compared to the more diffuse heating or cooling when $\lambda H>0$. The situation for $\lambda H<0$ is readily understood when one considers, for example, $\lambda<0, H>0$. Here we have a situation where the required heat transfer into the surface continuously lowers the thermal conductivity most significantly in the neighborhood of the body surface, thereby giving increasing resistance to the transfer of heat into the inner depths of the half-space. The result is that a larger and larger amount of the heat, which the body must continuously absorb, ends up being used primarily to increase the temperature of this confined neighborhood of the body 
surface; hence the catastrophic temperature rise at $x=0$. A similar argument for the case $\lambda H>0$ leads to the result of continuously increasing thermal conductivity at the surface region and correspondingly decreasing resistance to heat flow between the surface and the larger depths of the body; hence the more diffuse temperature distributions.

The technique of Meksyn for integrating the boundary layer equations of fluid mechanics has been successfully incorporated here in the solution to a problem in nonlinear heat diffusion. One would conclude that this technique in particular could be used as a basis for solving other more complicated problems of nonlinear diffusion, and that the understanding of such phenomena could be greatly enhanced by incorporation of previous successes of boundary layer research in general.

The author would like to express his thanks to Messrs. A. J. Schepis and W. W. St. Cyr for their interest and for the stimulating discussions engaged in throughout the course of this work.

\section{REFERENCES}

[1] D. Meksyn, New methods in laminar boundary layer theory, Pergamon Press, London, 1961

[2] J. Crank, Mathematics of diffusion, Oxford Press, London, 1956

[3] J. B. Rosser, Transformations to speed the convergence of series, J. Res. Nat. Bureau of Standards 46, 56-64 (1951)

[4] H. S. Carslaw and J. C. Jaeger, Conduction of heat in solids, Oxford Press, London, 1959 